\title{
Literarische Kunst und Friedensengagement in der Bombarda des Bartholomaeus Latomus
}

In seinem 2003 veröffentlichten Aufsatz zum Aristaeus-Epyllion in der neulateinischen Lehrdichtung präsentierte Heinz Hofmann auch eine reichhaltige Übersicht über neulateinische Lehrgedichte, die jenen Themen gewidmet sind, „die von den Alten noch nicht oder in den Augen der Humanisten nicht hinreichend behandelt worden waren, oder solchen Themen, die durch die wissenschaftlichen, technischen und gesellschaftlichen Entwicklungen der Neuzeit erst möglich wurden“. ${ }^{1} \mathrm{Zu}$ den technischen Entwicklungen gehört u. a. die Erfindung des Schießpulvers und der Feuerwaffen, und dementsprechend erscheint als diesbezügliches Beispiel das von Bartholomaeus Latomus verfasste Gedicht Bombarda. $^{2}$ Auch sonst wird dieses Gedicht in der modernen Forschung fast immer - und stets in unreflektierter Art und Weise - als Lehrgedicht bezeichnet, so etwa von IJsewijn und Sacré in ihrem Companion to Neo-Latin Studies ${ }^{3}$ und - auf deren Spuren - von Myriam Melchior in ihrer 2009 erschienenen Edition des Poems. ${ }^{4}$ Die wenigen früheren Gelehrten hingegen, die sich mit Latomus und seiner Bombarda beschäftigt haben, vermieden es interessanterweise, das Gedicht irgendeiner spezifischen Gattung zuzuordnen. ${ }^{5}$ Inwiefern ist eine

1 Heinz Hofmann: Aristaeus und seine Nachfolger. Bemerkungen zur Rezeption des AristaeusEpyllions in der neulateinischen Lehrdichtung. In: Humanistica Lovaniensia 52 (2003), S. 343398 (das Zitat S. 345).

2 Hofmann (Anm. 1), S. 349, Anm. 41.

3 Jozef IJsewijn, Dirk Sacré: Companion to Neo-Latin Studies. Second entirely rewritten edition. Part 2: Literary, linguistic, philological, and editorial questions. Leuven 1998 (Supplementa Humanistica Lovaniensia 14), S. 39.

4 Humanistica Luxemburgensia: La Bombarda de Barthélemy Latomus. Les Opuscula de Conrad Vecerius. Hg. von Myriam Melchior, Claude Loutsch. Bruxelles 2009 (Collection Latomus 321), S. 7-93, hier S. 16-17. Meine Zitate aus der Bombarda sind dieser Edition entnommen. Zu beachten ist allerdings die Rezension von Luc Deitz in: Bibliothèque d'Humanisme et Renaissance 73 (2011), S. 217-221.

5 Louis Roersch: Barthélemy Latomus, le premier professeur d'éloquence latine au Collège royal de France. In: Bulletins de l'Académie royale des Sciences, des Lettres et des Beaux-Arts de Belgique, 3e série, 14 (1887), S. 132-176; Eugen Wolff: Un humaniste luxembourgeois au XVIe siècle. Barthélemy Latomus d'Arlon (1498?-1570). Sa vie et son œuvre d'après des documents inédits. Première partie: 1498?-1541. Programm herausgegeben am Schlusse des Schuljahres 1901-1902. Großherzogliches Athenäum zu Luxemburg, Gymnasium. Luxembourg 1902; 
Charakterisierung der Bombarda als Lehrgedicht überhaupt vertretbar oder haltbar?

Bartholomaeus Latomus (ca. 1497/1498-1570) ${ }^{6}$ ist vor allem bekannt geblieben als erster lecteur royal für Latein am Kollegium, das der französische König François I. in Paris nach dem Modell des Leuvener Collegium Trilingue etabliert hatte. Vor dieser Ernennung, die 1534 auf Fürsprache von Guillaume Budé erfolgte, war er schon drei Jahre am renommierten Collège Sainte-Barbe als Dozent für Rhetorik tätig gewesen. Zuvor hatte Latomus in Freiburg, Trier, Köln und Leuven studiert und gelehrt. 1542 nahm der damalige Kurfürst und Erzbischof von Trier, Johann Ludwig von Hagen, ihn als Rat in Dienst. Bis zu seinem Tode arbeitete er im Umfeld der Trierer Erzbischöfe und erneuerte somit eine Verbindung, von der er auch schon während seines Studiums in Trier profitiert hatte. Außerdem ernannte Karl V. ihn 1548 zum Assessor am Reichskammergericht in Speyer. Sein Schrifttum weist ebenso wie seine Karriere ein doppeltes Bild auf. In der ersten Phase seiner Laufbahn, bis einschließlich seiner neunjährigen Pariser Zeit, profilierte er sich insbesondere als humanistischer Philologe und Literat und veröffentlichte neben einigen Dichtungen vor allem Schriften zur Rhetorik und Kommentare zu verschiedenen Werken Ciceros, die im Laufe des sechzehnten Jahrhunderts häufig nachgedruckt wurden. Während er in dieser Zeit durchaus einigen Aspekten der Reformation zugeneigt war, vertrat er seit seiner Rückkehr nach Trier in aller Klarheit die römisch-katholische Linie und engagierte sich mit mehreren Streitschriften in theologischen Kontroversen, die er u. a. mit Martin Bucer austrug.

Das Gedicht Bombarda ist in zwei Phasen zustande gekommen. Eine erste knappe Fassung erschien 1523 als Begleitstück zu einem längeren carmen historicum, der Factio memorabilis Francisci ab Siccingen cum Trevirorum obsidione, tum exitus eiusdem (Köln: Eucharius Cervicornus 1523), in der Latomus die Belagerung Triers im September 1522 durch den Reichsritter Franz von Sickingen und ihre Vorgeschichte sowie den Untergang und Tod Sickingens (1523) episch

\footnotetext{
Latomus: Deux discours inauguraux. Hg. von Louis Bakelants. Bruxelles 1951 (Collection Latomus 5); Guy Cambier: Études sur le XVIe siècle. Hg. von Jean Bingen. Bruxelles 1982, S. 85142. Ein detaillierter bio-bibliographischer Überblick wurde erstellt von Louis Bakelants (und nach seinem Tod zum Abschluss gebracht von Marie-Thérèse Lenger) in der Bibliotheca Belgica. Bibliographie générale des Pays-Bas. Neue Ausgabe hg. von Marie-Thérèse Lenger. Bd. 3. Bruxelles 1964, S. 678-747 und Bd. 6. Bruxelles 1970, S. 9-171; 217-250.

6 In der Forschungsliteratur wird immer noch häufig 1485 als Geburtsjahr angegeben, obwohl Percy S. Allen schon 1924 berechtigten Zweifel gegen diese Datierung erhob; siehe Opus epistolarum Desiderii Erasmi Roterodami. Hg. von Percy Stafford Allen, Helen Mary Allen. Bd. 5. Oxford 1924, S. 2.
} 
ausmalt. Diese Belagerung Triers schildert Latomus als Augenzeuge und bescheinigt somit implizit die Wahrhaftigkeit seines Poems: ${ }^{7}$

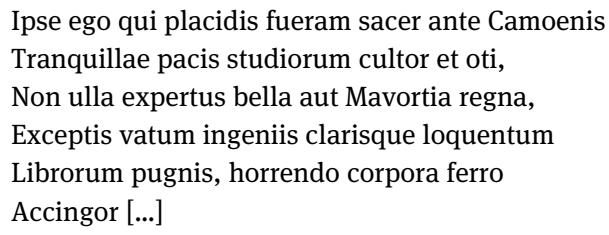

Schon in diesem Gedicht evoziert Latomus mehrmals und ausführlich den Beschuss mit Kanonen und ihre verheerende Gewalt. Somit bildet die Bombarda ein logisches Komplement: ${ }^{8}$ In einer prosopopoiia beschreibt sie selbst, mythologisch eingekleidet als Tochter Vulkans und der Erde (Tellus), ihre zerstörerische Kraft und erläutert knapp, auf welche Weise und mit welchen Materialien ihr Erfinder sie hergestellt hat - nicht ohne diesen dafür auch zu rügen.

Dieses poetische corollarium baute Latomus zu einem fast um das Zehnfache erweiterten Gedicht aus, das er 1536 unter demselben Titel in Paris erscheinen ließ. ${ }^{9}$ In dieser Form ist das Gedicht wohl das erste längere selbständige neulateinische Poem, das explizit der Kanone gewidmet ist. Zusammen mit dem Gegenstand tauchte auch der Begriff im vierzehnten Jahrhundert auf. ${ }^{10}$ Zunächst findet sich der Terminus in französischen und italienischen Chroniken, z. B. bei Jean Froissart und Giovanni Villani. Aus dem späten vierzehnten Jahrhundert stammt auch der erste lateinische Beleg in dieser Bedeutung (nicht

7 Factio memorabilis Francisci ab Siccingen cum Trevirorum obsidione, tum exitus eiusdem (Köln: Eucharius Cervicornus 1523), fol. cIVr-v. In der Staatsbibliothek zu Berlin - Preußischer Kulturbesitz befand sich bis zum Zweiten Weltkrieg ein Exemplar eines weiteren Druckes aus demselben Jahr (Soest: Nikolaus Schulting 1523); siehe Wolff (Anm. 5), S. 36, Anm. 4. Zu diesem Gedicht siehe Eckhard Bernstein: Franz von Sickingen's Revolt. The "Factio memorabilis Francisci ab Siccingen" (1523) by the Trier humanist Bartholomaeus Latomus and its historical background. In: Magister et amicus. Festschrift für Kurt Gärtner zum 65. Geburtstag. Hg. von Václav Bok, Frank Shaw. Wien 2003, S. 755-774.

8 Factio memorabilis (Anm. 7), fol. elIIv-fIVr.

9 Ad christianissimum Galliarum regem Franciscum Bartholomaei Latomi, Professoris eius in bonis literis Lutetiae, Bombarda. Eiusdem ad cardinalem Bellaium, episcopum Parisiensem, Elegiacon. Paris: François Gryphe 1536. Der Umfang der Bombarda stieg von 41 auf 389 Hexameter. Die Bibliothèque municipale in Beaune bewahrt ein seltenes Exemplar einer späteren Ausgabe, gedruckt 1545 in Paris bei Pierre Gromors.

10 Zum Folgenden siehe John Rigby Hale: Gunpowder and the Renaissance: an Essay in the History of Ideas. In: From the Renaissance to the Counter-Reformation. Essays in Honour of Garrett Mattingly. Hg. von Charles Howard Carter. London 1966, S. 113-144. 
,Blasinstrument'), im Kommentar des Benvenuto da Imola zu Dantes Inferno. ${ }^{11}$ Bekannt wurde der Terminus in der neulateinischen Literatur, als Lorenzo Valla und Bartolomeo Facio sich darüber stritten, ob die Vokabel ,bombarda‘ als neulateinischer Neologismus überhaupt akzeptabel sei. ${ }^{12}$ Seit dem fünfzehnten Jahrhundert wurde die Kanone als neue Feuerwaffe mehr und mehr thematisiert, sowohl in epischen und lyrischen Dichtungen als in der Historiographie. Generell wurden Feuerwaffen als unmenschlich und teuflisch betrachtet und dementsprechend negativ kommentiert, nicht zuletzt auch in moralischem Sinne: Feuerwaffen seien zwar effektiv, aber keine Waffen eines echten, heldhaften Kriegers. Für diese Einschätzung konnten frühneuzeitliche Autoren durchaus an antike Vorstellungen anknüpfen. So erzählte Ovid in seinen Fasti (1,571-572), wie Cacus, als dieser merkte, dass er in einem geregelten Zweikampf Hercules unterlegen blieb, zum Feuerspeien Zuflucht nahm. Dieses Kampfmittel hatte Cacus von seinem Vater Vulkan gelernt (patrias artes); es entlarvte ihn aber sogleich als einen Feigling (male fortis). ${ }^{13}$ Ein weiterer berüchtigter Flammenschleuderer war Salmoneus, der es wagte, Donner und Blitz nachzuahmen und sich auf diese Weise Juppiter gleichzustellen; für diesen Frevel wurde er in der Unterwelt bestraft (Vergil, Aeneis, 6,585-594).

Kritiker und Gegner von Feuerwaffen konnten sich aber nicht dauerhaft durchsetzen; die Effektivität dieser Waffen war zu evident. Nicht zuletzt bei deutschen Autoren machte sich schnell eine gewisse Faszination und ein technisches Interesse bemerkbar, gelegentlich sogar ein gewisser Nationalstolz, etwa bei Jakob Wimpfeling in seiner Epithoma Rerum Germanicarum: Aus seiner Sicht beweist die Erfindung der Kanone, dass ita non solum Germani nostri, vel ipsis exteris testantibus, bellatores semper fuisse acerrimi, sed instrumentorum quoque bellicorum inventores subtilissimi videntur. ${ }^{14}$ Gegenstimmen, wie

11 Johann Ramminger: bombarda. In: Ders., Neulateinische Wortliste. Ein Wörterbuch des Lateinischen von Petrarca bis 1700. www.neulatein.de/words/2/002722.htm (11. September 2019). Siehe auch die Belege im Artikel „Bombarda“ des Glossarium Mediae et Infimae Latinitatis. Hg. von Charles du Fresne, sieur du Cange, neueste Edition hg. von Léopold Favre. Bd. 1. Niort 1883-1887, S. 694-695.

12 Dieser Disput, der vor dem Hintergrund der humanistischen Debatte über neulateinische Neologismen zu verstehen ist, ist in der Forschungsliteratur häufig referiert worden. Siehe z. B. Ottavio Besomi: Dai ,Gesta Ferdinandi regis Aragonum“ del Valla al ,De orthographia‘ del Tortelli. In: Italia Medioevale e Umanistica 9 (1966), S. 75-121, hier S. 85 und 89-91.

13 Auf diese Ovid-Stelle machte mich Philip Schmitz während der Tagung dankenswerterweise aufmerksam.

14 Jakob Wimpfeling: Epithoma rerum Germanicarum usque ad nostra tempora. Straßburg: Johann Prüß d. Ä 1505, fol. 38v. 
jene von Konrad Celtis in seiner Ode 3,8 (Execrat Germanum inventorem bombardae, cuius pila paene traiectus fuisset), ${ }^{15}$ fielen dabei kaum ins Gewicht.

Inwiefern Latomus solche Belege kannte, ist schwer zu sagen. Auf neulateinische Texte nimmt er, soweit ich sehen konnte, keinen direkten Bezug. Vertraut dürfte er aber auf jeden Fall gewesen sein mit der Bombarda des Geschichtsschreibers und Dichters Pandolfo Collenuccio aus Pesaro. ${ }^{16}$ Es handelt sich hier um eine Fabel, in welcher der Autor die Entstehung der Kanone, genauer gesagt das physische Phänomen der Explosion in einer Kanone, allegorisch erklären will; hinter der Hauptfigur der Fabel, dem weisen Städtebauer Phronimus, der die von ihm gegründete Stadt mit Artillerie absicherte, verbirgt sich Collenuccios Schirmherr Ercole d'Este in Ferrara. Die Bombarda erschien postum 1511 bei Matthias Schürer in Straßburg mit einer Vorrede von Beatus Rhenanus an Jakob Spiegel, den Sekretär Kaisers Maximilian I. Dort sagt Beatus Rhenanus, dass die Bombarda Jakob Spiegel an die Belagerung Paduas (1509) zurückdenken lassen wird, wo die kaiserlichen Truppen mit Kanonen beschossen wurden. ${ }^{17}$ In ähnlicher Weise knüpfte Latomus als Autor an eine persönliche Erfahrung an, in seinem Falle in Trier.

Während aber die Fabel Collenuccios in aller Klarheit eine didaktische Intention des Autors zu erkennen gibt, ${ }^{18}$ ist eine vergleichbare Zielsetzung in Latomus' Bombarda nicht auf den ersten Blick sichtbar. Mehr noch, offensichtlich war es Latomus nicht daran gelegen, sein Gedicht eingangs als Lehrgedicht zu markieren. Die dazu üblichen , markers ${ }^{\text {19 }}$ fehlen allesamt: Latomus gibt keine detaillierte Inhaltsangabe, die den Aufbau und die Struktur des Gedichts offenlegt, entwickelt keine poetologische Reflexion, signalisiert keinen Rückgriff

15 Konrad Celtis: Oden / Epoden / Jahrhundertlied. Libri Odarum quattuor, cum Epodo et Saeculari Carmine (1513). Hg. von Eckart Schäfer. Tübingen 2008 (NeoLatina 16), S. 228-233. Dass der Erfinder der Kanone ein Deutscher sei, war eine in der Frühen Neuzeit weit verbreitete Erkenntnis, die sich aber aus heutiger Sicht nicht erhärten lässt.

16 Siehe Paolo Paolini: L'apologo latino ,Bombarda‘ di Pandolfo Collenuccio e altri riflessi letterari delle prime armi da fuoco. In: Respublica Litterarum 12 (1989), S. 155-163.

17 Pandolfo Collenuccio: Apologi quatuor. Agenoria. Misopenes. Alithia. Bombarda. Straßburg: Matthias Schürer 1511, fol. A2r: „Bombarda demum violenti tormentariarum machinarum impetus rationem ostendit; quem tu apologum legens recordabere creberrimorum iactuum, quibus Caesariani in Patavina obsidione impetebantur, ubi staturae brevitatem tibi plus semel saluti fuisse ferunt.“

18 Collenuccio äußerte sich auch selber zum didaktischen Zweck seiner Bombarda in einem Brief an einen Freund, der ihn um Erläuterung einiger schwierigen Passagen gebeten hatte; siehe Harold Andrew Mason: Wyatt and Hercules. In: ELH. Journal of English Literary History 51 (1984), S. 207-2018, hier S. 211.

19 Thomas Haye: Das lateinische Lehrgedicht im Mittelalter. Analyse einer Gattung. Leiden 1997 (Mittellateinische Studien und Texte 22), S. 168-223. 
auf ein Gattungsmodell des Lehrgedichts, entwirft keine Lehrer-Schüler-Konstellation, deutet nicht auf die poetische Umformung einer Prosavorlage hin, und kündigt keine Vermittlung von Sachwissen an.

Darüber hinaus standen Latomus auch keine konkreten lehrhaften Modelltexte in poetischer Form zur Verfügung. Für technische Gegenstände war natürlich sowieso keine antike literarische Vorlage vorhanden, und generell wurde die Kriegskunst nicht in der Lehrdichtung, sondern im Epos poetisiert. Ansonsten wurden technische Einzelheiten der Kriegsführung gerne auch ausgiebig beschrieben in der neulateinischen Groß- und Kleindichtung zu zeitgenössischen Belagerungen (vom Typus „De obsidione ...“). Latomus knüpfte eben an eine solche Belagerung an; Spuren einer Rezeption anderer derartiger Gedichte, die er hätte kennen können, ${ }^{20}$ haben sich aber nicht finden lassen.

Stattdessen fällt von Anfang an der stark appellative Charakter des Gedichts auf. Auch wenn Latomus in den ersten beiden Versen weitgehend die Anfangsverse der früheren Kurzfassung, inklusive die mythologische Abstammung der ,Bombarda‘, übernahm, führte er dennoch eine bezeichnende Änderung durch: Während er 1523 die ,Bombarda‘ selber zu Wort kommen ließ, richtete er sich im Dezember 1536 an den französischen König François I., dem er die neue Bombarda auch widmete. Im Laufe des Jahres 1536 hatte dieser die Herzogtümer Savoie und Piemonte besetzt und hoffte wohl, weiter in das strategisch sehr wichtige Herzogtum Mailand vorzustoßen; somit provozierte er eine direkte Konfrontation mit Kaiser Karl V., zu dessen Einflusssphäre diese Gebiete gehörten. Im April 1536 protestierte Karl V. in Rom vor dem versammelten Konsistorium in Anwesenheit des Papstes Paul III. gegen diesen Übergriff des französischen Königs; in seiner aufsehenerregenden Rede geißelte Karl natürlich auch die schändliche Allianz, die François I. 1534 mit den Türken geschlossen hatte. ${ }^{21}$ Durch eine Fülle von rhetorischen Fragen und Reflexionen verstärkt Latomus dauernd die appellative Ausrichtung seines Gedichts und lässt sie am Ende in eine Klimax münden. Denn im letzten Teil seiner Bombarda evoziert er die vielen Kriege, die die beiden Fürsten schon ausgefochten haben, und ruft François I. zum Schluss dazu auf, seinen Streit mit Karl aufzugeben und gemeinsam mit

\footnotetext{
20 Z. B. Ioannes Candidus Venlonensis: Super obsidione Venlonis soluta Ode saphica, Köln: Heinrich Quentel (Erben) 1512. Die Waffengewalt wird farbenreich evoziert; die ,bombarda“ wird auf fol. aIVr genannt.

21 Siehe etwa Karl Brandi: Kaiser Karl V. Bd. 1: Werden und Schicksal einer Persönlichkeit und eines Weltreiches. 7. Auflage. München 1964 (zuerst 1937), S. 313-315, sowie Bd. 2: Quellen und Erörterungen. München 1941, S. 258-260.
} 
ihm und allen christlichen Fürsten die auswärtige Drohung der Türken abzuwenden (371-377): $:^{22}$

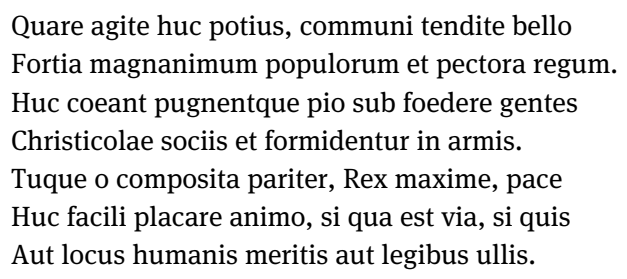

Diese unmittelbare politische Botschaft bildet gewiss das eigentliche Kernanliegen von Latomus' Bombarda. Schon in seinen früheren Dichtungen hatte er ein ähnliches politisches Engagement gezeigt und sich in ähnlichem Sinne an Karl V. und seinen Bruder Ferdinand gewandt. Mit diesem Plädoyer für Solidarität der christlichen Fürsten und einen gemeinsamen Krieg gegen die Türken lag Latomus ganz auf der Linie des Erasmus von Rotterdam. Die beiden kannten sich und schätzten sich gegenseitig. ${ }^{23}$ Schon 1518 , während seiner Lehrtätigkeit in Freiburg, könnte Latomus Erasmus in Basel begegnet sein (Epist. 3029,55-56); ${ }^{24}$ auf jeden Fall trafen sie sich während Erasmus' Reise nach Basel im November 1521 (Epist. 1342,212-214). ${ }^{25}$ Die prosopopoiia der ,Bombarda‘ 1523 könnte wohl durch das Auftreten der ,Pax“ in Erasmus' Querela Pacis inspiriert gewesen sein.

In der Langfassung seiner Bombarda unterstreicht Latomus e contrario seinen Friedensappell mittels der Evozierung von Kriegsgewalt durch Kanonen. Die Apostrophe an François I. am Anfang und der Appell am Schluss umrahmen im Sinne einer Ringkomposition drei etwa gleich lange Abschnitte, die alle in einem jeweils unterschiedlichen Register lehrhafte Züge aufweisen. Diese Segmente sind der Reihe nach (1) sachlich, (2) historisch und (3) mythologisch orientiert, werden aber alle dergestalt rhetorisch aufbereitet, dass sie die zerstörerische Vehemenz der Kanone anprangern und somit unterschwellig Latomus' Friedensdiskurs unterstützen.

22 Bombarda. Hg. Melchior (Anm. 4), S. 85.

23 Ilse Guenther, Peter G. Bietenholz: Bartholomaeus Latomus. In: Contemporaries of Erasmus. Hg. von Peter G. Bietenholz, Thomas B. Deutscher. Bd. 2. Toronto, Buffalo, London 1986, S. 303-304.

24 Opus epistolarum (Anm. 6), Bd. 11. Hg. von Helen Mary Allen, Heathcote William Garrod. Oxford 1947, S. 146.

25 Opus epistolarum (Anm. 6), Bd. 5. Oxford 1924, S. 208. 
(1) Zunächst beschreibt Latomus die Erfindung der Kanone und schickt diesem Bericht eine Invektive gegen den (in keinerlei Weise präzisierten) Erfinder dieser Feuerwaffe voraus (13-17): ${ }^{26}$

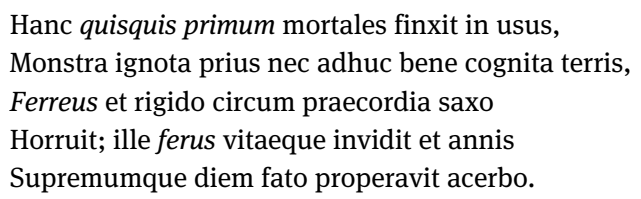

Tib., 1,10,1-2

Die Assoziation ferreus - ferus, die Latomus aus Tibulls Friedenshymnus $(1,10,2)$ entlehnt und die auf das eiserne Zeitalter und die damit einhergehende Pervertierung und Zerrüttung anspielt, ist das Leitmotiv der ganzen Tirade. Die darauffolgenden Verse bilden den einzigen Passus, in dem Latomus auf die Konstruktion der Kanone und die Herstellung des Schwarzpulvers eingeht. Die drei Bestandteile des Schwarzpulvers etwa, Schwefel (sulphur), Holzkohle (carbones) und Salpeter (nitrum), und seine Zündung beschreibt er folgendermaßen (77-80): ${ }^{27}$

Scilicet et foedo cum sulphure miscuit atros

Carbones viridisque horrentia pondera nitri

Pulvereasque armavit opes imamque sub alvum

Intulit et tenui flammam commisit hiatu.

Diese knappen technischen Angaben werden aber ganz in einer emotional geladenen Schilderung der katastrophalen Folgen der Anwendung eines solchen Geschosses eingebettet.

(2) Im zweiten Abschnitt berichtet Latomus über die Belagerung Triers durch Franz von Sickingen. Latomus' Zielrichtung bleibt aber dieselbe: Seine Narration dieser historischen Episode dient primär dazu, die verheerenden Folgen

26 Bombarda. Hg. Melchior (Anm. 4), S. 35-36. Hervorhebungen von mir. Hier wie auch sonst mehrmals greift Latomus auf Verse seiner Kurzfassung von 1523 zurück. Vergleiche Factio memorabilis (Anm. 7), fol. eIII v: „Qui me qui primum mortales finxit in usus | Invisumque instruxit opus docuitque parari, | Ferreus et rigido circum praecordia saxo | Obsitus ille fuit vitaeque invidit et annis.“

27 Bombarda. Hg. Melchior (Anm. 4), S. 47. Vergleiche Factio memorabilis (Anm. 7), fol. eIIIv-eIVr: „Scilicet et viridi squalentia sulphura nitro | Miscuit et pariter, carbonibus additis atris, | Pulvereasque armavit opes imamque sub alvum | Intulit et tenui flammam commisit hiatu.“ 
des Einsatzes von Kanonen offenzulegen. Auch hier wird der Tenor gleich beim Einstieg bestimmt (101-111): ${ }^{28}$

Vidi ego qua liquidum Trevir vetus accolit amnem
Quaque virum laeto miscentur flumina Rheno,
Flumina vitiferi semper mihi grata Mosellae,
Vidi hic ingentem conflato ex aere columnam,
Vas immane, stupent solo quod lumina visu,
Cum procul emissum duri sub vertice montis
Horrendo terram fremitu caelumque profundum
Complesset simul et foedo circum omnia odore
Saxa per et solidae rupisse obstantia cautis
Viscera, per mediam (dictu mirabile) rupem
Septenosque pedes montem penetrasse sub imum.

Wie in der Factio memorabilis erhöht Latomus auch hier die Überzeugungskraft seiner Darstellung durch einen Hinweis auf seine Autopsie. Er verwendet dafür die Junktur vidi ego, die als poetische Formel in der lateinischen Dichtung auch gerade am Anfang eines Verses - reichlich belegt ist, und zwar nicht nur in der historischen und didaktischen Epik, sondern auch in der Lyrik sowie in Komödien und Tragödien. Konkret beteuert er, ein „ungeheures Gefäß“ gesehen zu haben, „dort wo ein alter Trierer am klaren Fluss wohnt und wo dieser Strom sich mit dem Rhein, der sich über eine dichte Anwohnerschaft freut, vermischt, der mir immer angenehme Strom der Weinstöcke tragenden Mosel.“ Der Hinweis auf den vetus Trevir und seine idyllische Umgebung ließe sich vielleicht wie eine Anspielung auf den vergilischen senex Corycius (Georgica, 4,127) lesen. Allerdings ist nur die Autopsiebekundung ganz konkret mit der vergilischen Vorlage vergleichbar; die ganze praeteritio, in die Vergil seine Erinnerung (memini me vidisse) an den Korykischen Greis einbettet, wird von Latomus nicht nachgeahmt. ${ }^{29}$ Vielmehr kontrastiert er diesen malerischen Natureinstieg effektvoll mit dem Horror der Kanonengewalt, die unmittelbar darauf die Szenerie beherrscht

Die historischen Ereignisse werden als abschreckendes exemplum dargeboten und kommentiert. Allerdings lässt Latomus nicht nach, auch die Effektivität der neuen Artillerie in den Händen der französischen Armee zu betonen, z. B. bei der Eroberung von Turin oder der Verteidigung von Péronne (beide 1536).

28 Bombarda. Hg. Melchior (Anm. 4), S. 51-53.

29 Zur Rezeption dieser ,Gartenpraeteritio“ in der neulateinischen Lehrdichtung siehe Ruth Monreal: Vergils Vermächtnis: Die Gartenpraeteritio in den Georgica (4, 116-148) und Typen ihrer Rezeption im neulateinischen Lehrgedicht. In: Humanistica Lovaniensia 54 (2005), S. 1-47. 
Den Schlusspunkt dieses zweiten Teils bildet ein Vergleich mit einem Seesturm, der genauso rücksichtslos wie eine Kanone alles dahinrafft (198-207): ${ }^{30}$

Qualis in aequoreo fluctu, cum Iuppiter atra Involvit sub nube polum ventique minaces Incubuere mari tumido, furit aestus et undae Immanes pulsant scopulos; tum nubibus ignes Abrupti ingeminant [...],

Haud aliter gemino bombarda incensa furore Concitat horrendos motus et proelia miscet.
Stat. Theb., 5,362

Verg. Aen., 1,84; 1,107

Verg. Aen., 3,199

Während epische Züge sich durchaus auch schon vorher im Gedicht bemerkbar machen, bemüht Latomus im Topos des Seesturms natürlich vollends das epische Register und zeigt, dass er etwa die berühmten Seesturm-Szenen aus Vergils Aeneis (1,81-156 und 3,192-204) und Statius' Thebais (5,361-383) klar vor Augen hat.

(3) Über dieses epische Gleichnis schafft Latomus auch einen Übergang zum dritten Abschnitt seines Poems. Diesen beginnt er nämlich mit einem weiteren Gleichnis, diesmal mit dem Vulkan Ätna auf Sizilien. Dieses Gleichnis dient als Aufhänger für einen mythologischen Exkurs, in dem Latomus die Geschichte des Giganten Enceladus und der Gigantomachie ausführt. Das tertium comparationis ist erneut die explosive Wucht; in diesem Sinne wurden Vulkan und Kanone auch schon von früheren neulateinischen Autoren häufig aufeinander bezogen. Auch die von Latomus am Anfang seines Gedichts angedeutete Abstammung der ,Bombarda' von Vulkan und der Erde wird auf diese Weise kontextualisiert, denn auch die Giganten waren Söhne der Tellus; ,Bombarda‘ und die Giganten sind m.a.W. Geschwister. Latomus erzählt die Geschichte des Enceladus als aition für die Ausbrüche des Vesuvs und bezieht sich dafür hauptsächlich auf Vergils Beschreibung des Ätna in Buch 3,571-587 der Aeneis. Ähnlich wie Vergil erklärt Latomus die vulkanische Aktivität des Ätna mit der Wut des brennenden Enceladus, nachdem dieser von Juppiter niedergeschmettert und unter dem Ätna begraben worden war $(280-289):^{31}$

Hic igitur magnae imposito dum pondere molis

Urgetur grandesque illi angit anhelitus artus,

Nunc quoque sidereos, quos tunc conceperat, ignes

Sulphurea cum nube cavo de vertice montis

Eructat. Furit intus atroci murmure Tellus
Verg. Aen., 3,579-580

Verg. Aen., 3,576; 3,582

30 Bombarda. Hg. Melchior (Anm. 4), S. 65. Hervorhebungen von mir. 31 Bombarda. Hg. Melchior (Anm. 4), S. 75. Hervorhebungen von mir. 
Conclusumque premens violento carcere nimbum Ingentes cumulat vires maiusque premendo

Cogit onus, donec ruptis fornacibus altos

Verg. Georg., 1,472; Aen., 3,580

Flammarumque globos liquefactaque pondera rupis

Eiicit aethereas rauco stridore sub auras.

Verg. Aen., 3,574; Georg., 1,473

Verg. Aen., 3,576

Die beiden narrativen Einschübe, der eine historisch, der andere mythologisch, werden - wie gesagt - in epischem Stil ausgemalt. Solche epischen Einlagen sind an sich für das Lehrgedicht charakteristisch; das kanonische Modell hierfür war das Aristaeus-Epyllion in Vergils Georgica $(4,317-558) .{ }^{32}$ Es ist aber sehr bezeichnend, dass Latomus für seine Darstellung eine einschlägige Passage aus Vergils Aeneis und sonst nur den damit verwandten kürzeren Passus aus den Georgica (1,471-473) ausschlachtet. Andere, weit ausführlichere Texte aus der römischen Dichtung, die über den Vulkan Ätna als Naturphänomen handeln, insbesondere ein Passus aus Lukrezens De rerum natura $(6,639-702)$ und das anonym überlieferte Aetna-Gedicht aus der Appendix Vergiliana, werden kaum herangezogen. Vor allem übernimmt Latomus nicht die Pose des Lehrdichters, die er sowohl im Aetna-Gedicht als auch im Aetna-Passus bei Lukrez finden konnte (De rerum natura, 6,639-641): Nunc ratio quae sit, per fauces montis ut Aetnae / Exspirent ignes interdum turbine tanto, / Expediam. Zur Lehrdichtung bekennt sich Lukrez auf diese explizite Weise übrigens noch einmal im Aetna-Abschnitt (De rerum natura, 6,680-682), und auch mehrmals sonst in De rerum natura, insgesamt elfmal allein schon mit diesem spezifischen Verb.

Latomus nimmt - wohl bewusst - auf diese Passagen keinen Bezug. Allenfalls schimmert neben dem rein strukturellen Merkmal der narrativen Einlage eine weitere Charakteristik des Lehrgedichtes, die Latomus auch in Vergils Georgica wahrnehmen konnte, in der Bombarda durch, nämlich die Anthropomorphisierung. Tiere und Naturelemente wurden in der Lehrdichtung gerne als Menschengestalten dargestellt. Die Bienen im 4. Buch der Georgica bilden ein eloquentes und einflussreiches Beispiel: Die von Vergil beschriebene Bienengemeinschaft funktioniert wie die Gesellschaft der Menschen, ist ein Abbild in Miniatur des Menschenstaates. Demgemäß wird auch bei den Bienen das harmonische Zusammenleben manchmal durchkreuzt von Streit und Krieg, wie wenn zwei Könige um die Vorherrschaft kämpfen (Georgica, 4,67-94). In ähnlicher Weise modelliert Latomus die Kanone an einigen Stellen zu einer belebten, monströsen Kreatur, ${ }^{33}$ etwa im oben zitierten Gleichnis mit dem Seesturm.

32 Zur Rezeption des Aristaeus-Epyllion in der neulateinischen Lehrdichtung siehe Hofmann (Anm. 1).

33 Dies bemerkte schon Wolff (Anm. 5), S. 75. Latomus greift damit auch die Personifizierung der ,Bombarda‘ als Sprechinstanz in seiner Kurzfassung auf. 
In den narrativen Teilen seiner Bombarda will Latomus sich nicht als Lehrdichter profilieren, sondern als ein Dichter, der sich für diese Abschnitte von der römischen Epik inspirieren lässt, genauso wie er schon mit der Factio memorabilis an die historische Kleinepik angeknüpft hatte. In allen drei zentralen Teilstücken seines Gedichts thematisiert er aus einem jeweils unterschiedlichen Blickwinkel das Vernichtungspotential der Kanone und warnt somit implizit vor den gravierenden Folgen, die der Einsatz dieser Waffe nach sich zieht. Diese sachlich, historisch und mythologisch untermauerte Warnung kulminiert im Schlussteil in einem Aufruf an François I., diese Waffe nur dort einzusetzen, wo sie aus Latomus' Sicht alleinig sinnvoll ist, nämlich bei der Abwehr einer auswärtigen Drohung, die das gesamte christliche Europa existentiell gefährdet und daher gemeinsam mit dem römisch-deutschen Kaiser abgewehrt werden soll (381-389): ${ }^{34}$
Ambo animis studiisque pares pariterque verendi
Parcite fraternis manibus, discedite castris
Luctificis tristesque alio divertite pugnas!
Horreat et latis Asiae qui regnat in oris
Turca minax, vestro metuat concurrere ferro
Aut occumbat eo! Vestro sub Marte triumphent
Fulgida signa Crucis, quorum pia numine virtus
Vivat et o positis concordia floreat armis
Externumque ferox Bombarda feratur in hostem.

So beendet Latomus seine Bombarda mit einer klaren Handlungsanweisung an den Widmungsträger des Gedichts. Dahinter steht eine politische Moraldidaxe, die gut in einen Fürstenspiegel passen würde. In diesem Kontext erhalten auch die lehrhaften Ausführungen in den drei mittleren Teilen des Gedichts ihren eigentlichen Sinn. Sie sollen letztlich François I. dazu bringen, Latomus' Aufforderung stattzugeben. Somit ist die Bombarda nicht so sehr Lehrdichtung, sondern vielmehr Mahndichtung: Latomus' Ziel ist nicht so sehr docere, sondern vielmehr movere; seine Lehre wird zur Belehrung. Mit seiner Bombarda beteiligt Latomus sich, ebenso wie mit seinen früheren Dichtungen, im Sinne eines rhetorisch ausgeformten Friedensappells an einer aktuellen politischen Diskussion. Während er dafür vorhin Formen der Kasualdichtung in Anspruch genommen hatte, entschloss er sich nun, zu diesem Zweck strategisch ausgewählte Charakteristika der Lehrdichtung zu instrumentalisieren.

34 Bombarda. Hg. Melchior (Anm. 4), S. 87. 
Als Lehrgedicht wird man Latomus' Bombarda kaum bezeichnen können, es sei denn, man bezieht sich auf eine Variante, die meistens aus dem Rahmen des antiken Lehrgedichts verbannt wird, aber gerade durch eine ähnliche Verbindung von Lehrhaftigkeit und Paränese geprägt wird, nämlich das sogenannte ,christliche Lehrgedicht', wie die Instructiones Commodians, das Commonitorium des Orientius oder Prudentius' Apotheosis und Hamartigenia. Alle diese Lehrgedichte heben sich von den heidnisch-klassischen Modellen nicht nur durch eine veränderte Thematik ab, sondern ebenso durch das leidenschaftliche und missionarische Engagement, mit dem der jeweilige Autor seine Leserschaft $\mathrm{zu}$ einer bestimmten Lebensführung mahnen will. In der heidnischantiken Tradition ist diese Tendenz nur wenig ausgeprägt; sie macht sich allenfalls in Lukrezens De rerum natura einigermaßen bemerkbar. Inwiefern solche Poeme sinnvoll in die Geschichte des antiken Lehrgedichts und seiner nachantiken Wirkung integriert werden können, ist im jetzigen Stand der Forschung noch eine weitgehend offene Frage. ${ }^{35}$ Latomus selber suggeriert in keinerlei Weise, dass er diese spezifisch christliche Variante des Lehrgedichts $\mathrm{zu}$ rezipieren versucht habe. Ob man seine Bombarda dennoch als Lehrdichtung oder lehrhafte Dichtung deuten kann, hängt wohl von der Frage ab, wie viel Platz man der Paränese - neben der reinen Vermittlung von Fachwissen - im Verständnis von Lehrdichtung einzuräumen bereit ist.

Etwa anderthalb Jahrhunderte nach Latomus widmete der französische Jesuit François Tarillon (1666-1735) dem Schießpulver ein Gedicht. Sein Pulvis Pyrius. Carmen erschien zuerst 1692 in Paris (apud viduam Simonis Benard)

35 Während Bernd Effe in seiner bedeutsamen Monographie zur gesamten antiken Lehrdichtung die spätantike christliche Ausprägung explizit ausklammert (Dichtung und Lehre. Untersuchungen zur Typologie des antiken Lehrgedichts. München 1977 (Zetemata 69), S. 36), weist Thomas Haye hingegen in seiner Gattungsmonographie zum mittellateinischen Lehrgedicht (Anm. 19) auf einige deutliche Verbindungen (neben ebenso klaren Unterschieden) zwischen den ,kanonischen“ römischen Modellschriften (Lukrezens De rerum natura, Vergils Georgica und Manilius' Astronomica) und den christlichen Lehrgedichten der Spätantike hin und spricht vorsichtig von einer „Grenzzone der Gattung“ (S. 359-360, das Zitat S. 360). Während eine gewisse, nach Manilius eintretende Zäsur in der Tradition des Lehrgedichtes nicht zu übersehen ist, wie Claudia Schindler verdeutlicht und mit überzeugenden Gründen erklärt (Vom Kochrezept zu den Sternen: Aspekte der Gattungsgenese und Gattungsentwicklung im römischen Lehrgedicht. In: Wissensvermittlung in dichterischer Gestalt. Hg. von Marietta Horster, Christiane Reitz. Stuttgart 2005 [Palingenesia 85], S. 193-209), stellt das christliche Lehrgedicht dennoch keinen völligen Neustart dar, und daher sollte wohl nicht von einem radikalen Bruch ausgegangen werden. Wolfgang Schmid nimmt die „christlichen Lehrgedichte“ als eigene Rubrik in seinen Überblicksartikel zum Lehrgedicht (in: Lexikon der Alten Welt. Zürich 1965, Sp. 1699-1703, hier Sp. 1703) auf und unterscheidet zwischen einer „ethisch-paränetischen“ und einer „antihäretisch-dogmatischen“ Variante. 
und wurde in die maßgebliche Sammlung von Poemata didascalica seines Ordensgenossen François Oudin aufgenommen. ${ }^{36}$ Ein Vergleich dieses Gedichts mit Latomus' Bombarda macht sowohl Ähnlichkeiten als auch Unterschiede sichtbar und zeigt, wie viele Schattierungen die Lehrhaftigkeit in der Lehrdichtung annehmen kann. Genauso wie Latomus baute auch Tarillon eine mythologische Episode mit aitiologischer Intention in sein Poem ein: Den Ursprung des Schießpulvers führt er auf die sizilischen Zyklopen zurück, die Vulkan unter dem Ätna erstmals in die Kunst, mit Schwefel explosive Blitze zu schmieden, einweihte, und zwar aus Wut über seine Verbannung aus dem Olymp: ${ }^{37}$

Ille dolore furens amissique aetheris ira

Aetnaeo Siculos - perhibent - Cyclopas in antro

Primus fulmineos imitari sulphure motus

Edocuit, ruptis hinc Aetna effusa caminis

In superos; tum saevae artes, tum cognita primum

Attonitas late turbarunt murmura gentes.

Ebenso verweist Tarillon auf einen aktuellen militärischen Konflikt, nämlich die Feldzüge des Louis XIV. und konkret dessen Eroberung von Namur 1692, ${ }^{38}$ durch welche die destruktive Gewalt der modernen Feuerwaffen noch einmal illustriert wird. Anders als Latomus aber konstruiert Tarillon diesen Verweis als eine Schlussbetrachtung des Pulvis Pyrius: ${ }^{39}$

Haec super igniferis audaci carmine nitris Verg. Georg., 4,559

Ludebam, late Europam dum Francica in omnem

Nitra tonant, regum quo tempore iura tuetur

Accinctus flammis Lodoix validasque Namurci

Arces et pavidos mittit sua sub iuga Belgas.

Dieser autobiographische Ausblick ist insgesamt der abschließenden Sphragis von Vergils Georgica (4,559-566) nachgebildet. Dementsprechend markiert Tarillon in aller Deutlichkeit sein Carmen als Lehrgedicht, und somit fungiert auch sein mythologischer Exkurs ebenfalls als Gattungssignal. Außerdem leitet Tarillon sein Gedicht in typischer, wiederum durch Vergils Georgica kanonisierter Manier

36 François Oudin: Poemata didascalica. Bd. 1. Paris 1749, S. 128-140; ebd. Bd. 1. Paris 1813², S. $117-128$.

37 Oudin 1749 (Anm. 36), S. 139. Siehe auch Yasmin Annabel Haskell: Loyola’s Bees. Ideology and Industry in Jesuit Latin Didactic Poetry. Oxford 2003, S. 124.

38 Dieser Eroberung hat Tarillon ein eigenes Gedicht gewidmet: Namurcum a Ludovico Magno expugnatum. Paris: Witwe des Simon Bénard 1692.

39 Oudin 1749 (Anm. 36), S. 140. Hervorhebung von mir. 
ein, indem er die verschiedenen Facetten seines Themas in aufeinander folgenden indirekten Fragen auflistet: ${ }^{40}$

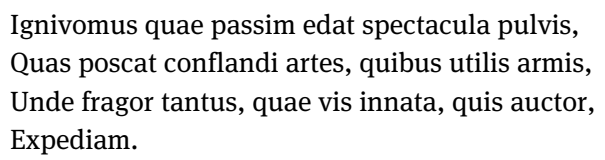

Daran schließt sich, genauso wie bei Vergil, eine Apostrophe an die einschlägigen Gottheiten an, zu denen sich im Pulvis Pyrius Louis XIV. als Widmungsträger gesellt. Tarillon lässt daher keinen Zweifel daran bestehen, dass er sein Poem in die Gattungstradition des Lehrgedichts einreihen wollte. Latomus' Bombarda ist viel weniger leicht $\mathrm{zu}$ bestimmen und kann wohl kaum einer spezifischen Gattung zugeordnet werden. Erschwerend kommt noch hinzu, dass der Terminus ,Lehrgedicht‘ generell etwas irreführend ist, da er nicht alle Aspekte dieses Texttypus abdeckt. Es geht im Lehrgedicht nicht ausschließlich um Lehre im Sinne von Vermittlung von Fachwissen: Zum einen beschränkte sich schon in der Antike, wie oben angemerkt, die auktoriale Sprechinstanz nicht notwendigerweise auf eine sachliche Unterrichtung, zum anderen wurde die poetische Form nicht selten einfach als Mehrwert einer inhaltlichen Botschaft verstanden. Dementsprechend sind die Konturen dieser Gattung im Laufe ihrer Geschichte immer wieder aufgeweicht worden. ${ }^{41}$ Dass die Bombarda von der Forschung als Lehrgedicht bezeichnet werden konnte, ist wohl nicht (lediglich) auf eine unsorgfältige Lektüre zurückzuführen, sondern auch durch die Proteusartige Natur der Lehrdichtung selbst zu erklären.

40 Oudin 1749 (Anm. 36), S. 128.

41 Siehe etwa die vielen „Abgrenzungen und Übergänge“, die Thomas Haye (Anm. 19), S. 242-298, für das mittellateinische Lehrgedicht auslotet. Wilhelm Kühlmann macht darauf aufmerksam, dass der Begriff ,Lehrdichtung، ein ,jüngeres wissenschaftliches Abstraktum für ein traditionsreiches Konzept“ ist (Wissen als Poesie. Ein Grundriss zu Formen und Funktionen der frühneuzeitlichen Lehrdichtung im deutschen Kulturraum des 16. und 17. Jahrhunderts. Berlin, Boston 2016 [Frühe Neuzeit 204], S. 1, Anm. 3); übrigens hat der Terminus ,Lehrdichtung/Lehrgedicht‘ keine wortwörtliche Entsprechung in anderen modernen Sprachen. Die von Kühlmann vorgeschlagene Definition der Lehrdichtung (ebd., S. 1-2: „das versgebundene, mehr oder weniger ästhetisch ambitionierte Schrifttum zur Vermittlung oder poetischen Nobilitierung von Sach-, Verhaltens- und Orientierungswissen“) umfasst sinnvollerweise mehrere Schattierungen des Lehrhaften in der Lehrdichtung. Zustimmend auch Jörg Robert, der zugleich vor einer zu eng fixierten Definition der Lehrdichtung warnt (Poetische Naturwissenschaft. Martin Opitz' Lehrgedicht Vesuvius [1633]. In: Daphnis 46 [2018], 188-214, hier S. 192, Anm. 11): „Weniger denn je hilft daher der Gattungsdiskussion ein normativ-begriffsrealistischer Zugriff, der analytische Kategorien in Reinform postuliert, wo die imitatorische Praxis durch Leitzitat und Motivkorrespondenz spontan Fährten der Identifikation legt.“ 
